\title{
Chemische Untersuchungen der Zăhne.
}

\author{
II. Teil.
}

Von

Th. Gaßmann.

(Der Redaktion zugegangen am 5. November 1909.)

Behufs Erweiterung meiner Forschung nach den Ursachen der Zahnkaries unterzog ich, anschließend an meine Publikation über chemische Untersuchungen der Zähne, ${ }^{1}$ ) prähistorische Menschenzähne der chemischen Analyse. Es war mir ein Bedürfnis, zu wissen, ob zwischen prähistorischen Menschenzähnen und solchen der Jetztzeit Unterschiede in ihrer chemischen Zusammensetzung bestehen und wenn ja, ob dieselben Rückschlüsse auf die Zahnverderbnis unserer Zeit herleiten lassen. Die mir gestellte Aufgabe wurde mir dadurch erleichtert, daß die Direktion des schweizerischen Landesmuseums in Zürich in höchst entgegenkommender und dankeswerter Weise mir prähistorische Menschenzähne aus ihrer Sammlung zur Verfügung stellte. Es waren dies Zähne, welche bei Ausgrabungen in Frauenfeld, Schweiz, gefunden wurden und aus den Jahren 200-50 v. Chr. datieren. Das Glück war mir insofern hold, als sich unter den mir zugekommenen Exemplaren auch Eckzähne befanden, wodurch der Vergleich der analytischen Daten von prähistorischen Menschenzähnen und solchen der Jetztzeit nur um so getreuer ausfallen konnte.

Die Reinigung der Zähne von zufällig anhaftenden, nicht zu den Zahnbestandteilen gehörenden Stoffen geschah in üblicher Weise mittels Wasser und Äther. Dabei erhielten die Zähne ein gelbes Aussehen, einen Glanz zeigend, der lebhaft an denjenigen von Hundezähnen erinnert. Die Vermutung, daß eventuell Zersetzung bei diesen Individuen stattgefunden habe, trifft in keiner Weise zu, im Gegenteil waren dieselben trotz ihres 2000 Jahre langen Verharrens in der Erde durchaus von keiner Beschädigung oder Veränderung befallen.

1) Chemische Untersuchungen der Zähne von Th. Ga ßm ann, Zürich. Diese Zeitschrift, Bd. LV, Heft 6. 
Die fein gepulverte und bei $110-120^{\circ}$ bis zur Konstanz getrocknete Zahnsubstanz ergab nunmehr folgende analytische Werte (die Analysen wurden analog den früheren ausgeführt):

\section{Menschenzähne (prähistorische).}

Eckzähne (bleibende).

I. Analyse.

0,6359 g Zahnsubstanz gaben 0,0538 $\mathrm{g} \mathrm{H}_{2} \mathrm{O}=8,46 \% \mathrm{H}_{2} \mathrm{O}$.

$0,2341 \mathrm{~g}$ Zahnsubstanz gaben $0,0583 \mathrm{~g}$ Glühverlust $=24,90 \%$

0,2341 » » $0,0982 » \mathrm{CaO} \quad \mathrm{Ca}=29,94 \%$

0,2341 » » $0,0011 * \mathrm{MgO} \mathrm{Mg}=0,28 \%$

$0,2341 \gg \quad \gg 0,0692 * \mathrm{P}_{2} \mathrm{O}_{5} \quad \mathrm{PO}_{4}=39,00 \%$

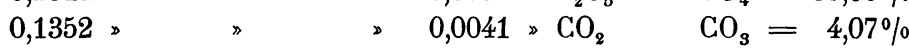

0,1740 » » 0,0006 . $\mathrm{Cl}=0,34 \%$

0,3345 » » $0,0068 \gg \mathrm{K}_{2} \mathrm{PtCl}_{6} \mathrm{~K}=0,32 \%$

0,3345 » $\gg 0,0072 * \mathrm{Na}_{2} \mathrm{SO}_{4} \mathrm{Na}=\frac{0,68 \%}{99,53 \%}$

II. Analyse.

0,5218 g Zahnsubstanz gaben 0,0415 $\mathrm{g} \mathrm{H}_{2} \mathrm{O}=7,95 \% \mathrm{H}_{2} \mathrm{O}$.

$0,2423 \mathrm{~g}$ Zahnsubstanz gaben $0,0618 \mathrm{~g}$ Glühverlust $=25,50 \%$

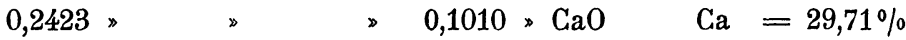

0,2423 " " 0,0014 " $\mathrm{MgO} \quad \mathrm{Mg}=0,34 \%$

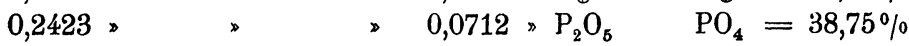

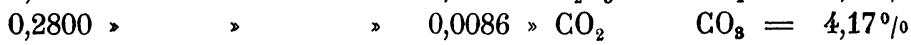

0,1500 » \ $\quad 0,0006$ " $\mathrm{Cl}=0,40 \%$

0,1582 * $\gg 0,0025 * \mathrm{~K}_{2} \mathrm{PtCl}_{6} \mathrm{~K}=0,25 \%$

0,1582 * " $0,0028 \times \mathrm{Na}_{2} \mathrm{SO}_{4} \mathrm{Na}=\frac{0,56 \%}{99,68 \%}$

Zur Erleichterung der Übersicht lasse ich hier anschließend die analytischen Daten von Eckzähnen unserer Jetztzeit folgen:

Menschenzähne (der Jetztzeit).

Eckzähne (bleibende). $8,09 \% \quad \mathrm{H}_{2} \mathrm{O}$.

\begin{tabular}{lr} 
Glühverlust & $=22,2 \%$ \\
$\mathrm{Ca}$ & $=29,78 \%$ \\
$\mathrm{Mg}$ & $=0,87 \%$ \\
$\mathrm{PO}_{4}$ & $=40,98 \%$ \\
$\mathrm{CO}_{3}$ & $=4,18 \%$ \\
$\mathrm{Cl}$ & $=0,41 \%$ \\
$\mathrm{~K}$ & $=0,34 \%$ \\
$\mathrm{Na}$ & $=0,61 \%$ \\
& \\
\cline { 2 - 2 } & $99,37 \%$
\end{tabular}


Ein Vergleich der Analysen von prähistorischen Menschenzähnen mit solchen von Zähnen unserer Jetztzeit belehrt uns sofort, daß recht ansehnliche Differenzen in ihrer chemischen Zusammensetzung bestehen. Es betrifft dies vor allem den Gehalt an Glühverlust, bezw. an organischer Substanz. Wir haben gemäß meiner früheren Publikation gesehen, daß die Hundezähne davon $26 \%$ aufweisen, die prähistorischen Menschenzähne $25 \%$ und unsere heutigen Zähne im Mittel $22 \%$. Wenn nunmehr die Hundezähne, die als die widerstandsfähigeren gelten, den höheren Gehalt an organischer Substanz voraus haben, wenn die prähistorischen Menschenzähne in dieser Beziehung den Hundezähnen ganz nahe kommen, so müssen wir zum mindesten annehmen, daß unsere jetzigen Zähne infolge ihres bedeutenden Rückstandes an organischer Substanz eine Veränderung erlitten haben, und wenn die Höhe des Glühverlustes für die Dauerhaftigkeit der Zähne spricht, so ist es einleuchtend, daß die Zähne dieser vor Christus lebenden Menschen widerstandsfähiger gewesen sind als unsere jetzigen.

Anders liegen die Verhältnisse beim Kalk; mit einer überraschenden Ähnlichkeit stimmt der Kalkgehalt der prähistorischen Menschenzähne mit demjenigen unserer jetzigen Zähne überein, damit mit Sicherheit dartuend, daß in diesem Punkte absolut keine Veränderung eingetreten ist. Da der Gehalt an organischer Substanz eine wesentliche Steigerung erfahren, der Kalkgehalt sich aber gleich geblieben ist, so war die Möglichkeit vorauszusehen, daß andere Bestandteile der Zähne in ihren Mengenverhältnissen zurückgedrängt werden.

Und in der Tat trifft dies zu und zwar zeigt eine bedeutende Verringerung des Gehaltes die Magnesia, gegenüber dem Bestand bei unseren jetzigen Zähnen eine Einbuße von etwa $60 \%$. Weniger auffallend tritt dies bei der Phosphorsäure zutage, die etwa $1-2 \%$ an Wert verliert.

\section{Zusammenfassung der Resultate.}

Zwischen prähistorischen Menschenzähnen und solchen unserer Gegenwart bestehen teilweise wesentliche Unterschiede.

Erstere besitzen größeren Gehalt an organischer Substanz, 
im Mittel $3 \%$, dafür vermindert sich ihr Gehalt an Magnesia und in geringerem Maße derjenige der Phosphorsäure.

Von eminenter Wichtigkeit ist, daß die Kalkmenge sowohl in den prähistorischen wie in unsern jetzigen Zähnen die gleiche geblieben ist, womit klar und deutlich nachgewiesen ist, daß unser Organismus, bezw. das Knochengewebe der Zähne keine Tendenz besitzt, seinen Kalkgehalt zu vermindern.

Ebenfalls ohne Veränderung des Gehaltes sind Kohlensäure, Natrium, Kalium und Chlor geblieben.

Zwischen Kalk und Wasser treten gewisse Beziehungen zutage; liegt der Wert des Kalkes zwischen 29,5-30\%, so bewegt sich der Wassergehalt immer zwischen $8-8,7 \%$, steigt nun ersterer an Gehalt, so geht letzterer zurück und umgekehrt.

In meiner früheren Arbeit habe ich bereits, gestützt auf die Analysen von Hundezähnen, darauf aufmerksam gemacht, daß der größere Gehalt an organischer Substanz bei der Dauerhaftigkeit der Zähne eine Rolle spielt, hier liegt es mir ob, nachdrücklich darauf hinzuweisen, daß die Analysen der prähistorischen Menschenzähne meine Depositionen von früher bestätigen, das heißt, sie sagen uns, daß unsere jetzigen Zähne in ihrer chemischen Zusammensetzung eine Änderung erfahren. $\mathrm{Daß}$ solche nicht ohne Einfluß auf die Lebensfähigkeit des Zahngewebes sein kann, liegt auf der Hand, um so mehr als die Wahrscheinlichkeit sehr nahe liegt, daß auch in Zukunft dieser Prozeß nicht innehält, sondern sich eher noch verschärft. 